\title{
Kinetic mechanism for water in vibrating carbon nanotubes
}

\author{
Xiaoyan Zhou ${ }^{1,2, *}$ and Fangqiang $\mathrm{Zhu}^{2, \dagger}$ \\ ${ }^{1}$ Department of Physics, Zhejiang Normal University, Jinhua 321004, China \\ ${ }^{2}$ Department of Physics, Indiana University-Purdue University Indianapolis, Indiana 46202, USA
}

(Received 6 April 2018; published 13 September 2018)

\begin{abstract}
Recent simulations revealed that, when an atom in a single-wall carbon nanotube was artificially driven to oscillate radially with the two ends of the nanotube fixed, water transport became highly unusual at some oscillation frequencies. Here we systematically investigate the underlying mechanism for such effects through a series of simulations and detailed analysis. We find that the pattern and magnitude for the vibration of the nanotube are sensitive to the driving frequency but largely independent of the presence of water. At certain resonance frequencies, some carbon atoms of the nanotube oscillate at much larger amplitudes than does the driving atom. Furthermore, a strongly vibrating nanotube tends to have a much-reduced water occupancy, which is mainly due to the heating effect rather than the induced deformation. Indeed, the water molecules inside the nanotube can be significantly heated and gain large kinetic energies due to the collisions with the vibrating carbon atoms. Consequently, the kinetic rate of water exchange through the nanotube could be enhanced even when the water occupancy is low. Our findings here may help understanding the physical mechanisms of similar nanodevices.
\end{abstract}

DOI: 10.1103/PhysRevE.98.032410

\section{INTRODUCTION}

The dynamic properties of water molecules in nanochannels are fundamental to understand the mechanism of water transport through biological channels and nanofluidic devices [1-4]. Numerous simulations of water confined in or transported across nanopores have shown many interesting properties of water dynamics, including transport rate [5-11], phase transitions [12-15], and gating behaviors [16-19] as in some biological channels $[2,20]$. These dynamic properties at nanoscales are the basis for various nanofluidic devices that have been proposed for their potential application in a variety of fields, such as desalination [21,22], energy conversion [23-26], and drug delivery [27].

It is well known that nanoscale systems may have unconventional properties and behaviors in comparison with bulk systems. In 2001, Hummer et al. found in molecular dynamics (MD) simulations that water molecules form an ordered single file inside a hydrophobic narrow carbon nanotube (CNT) and exhibit fast kinetics [12], which was described by a continuous-time random-walk model [28]. Fast liquid flow through CNT membranes was indeed reported later in experiments $[7,8]$. For narrow CNTs, water transport may significantly deviate from the prediction of continuum fluid dynamics, as shown by Thomas and McGaughey by using MD simulations [10]. Bocquet and coworkers carried out a series of theoretical and experimental works focused on the interfacial friction between water and the CNT wall [29-32] and found that the friction coefficient exhibits a strong dependence on curvature and becomes very small for

\footnotetext{
*zxylu@zjnu.cn

†fzhu0@iupui.edu
}

narrow armchair CNTs [29], which is consistent with the fast water transport through CNT membranes observed in experiments.

Nonequilibrium vibrations due to active driving forces may also play a nontrivial role in nanoscale systems and affect the transport properties of biological channels [33-35]. In the presence of active energy sources, the dynamic behavior of water confined in CNTs has been simulated in recent years [36-41]. Active transport of gas [36] and water [39] molecules through vibrating carbon nanotubes has been reported. In our previous work, we simulated a single-wall CNT when one of its atoms or an external charge was driven to oscillate [42-45]. Interestingly, water in the CNT exhibits ultrafast kinetics when the driving atom oscillates at certain frequencies. However, the underlying reason for the enhancement of water transport under such conditions has not been clearly understood.

In this study, we employ a series of MD simulations with detailed analysis to reveal the mechanism that relates the dynamics of water molecules in the CNT to the oscillation frequency of the driving atom.

\section{METHODS}

The simulation system is shown in Fig. 1. An uncapped, single-wall CNT $2.21 \mathrm{~nm}$ in length and $0.81 \mathrm{~nm}$ in diameter is embedded in the $z$ direction between two carbon sheets, which form an artificial membrane separating the bulk water on the two sides. Similar to earlier studies [45], we directly control the motion of one carbon atom in the CNT. Unlike the previous studies [45], however, here the chosen atom (Fig. 1) is exactly in the central plane, thus preserving the twofold symmetry of the CNT along the axial $(z)$ direction. In each simulation, this carbon atom was forced to undergo perfect 


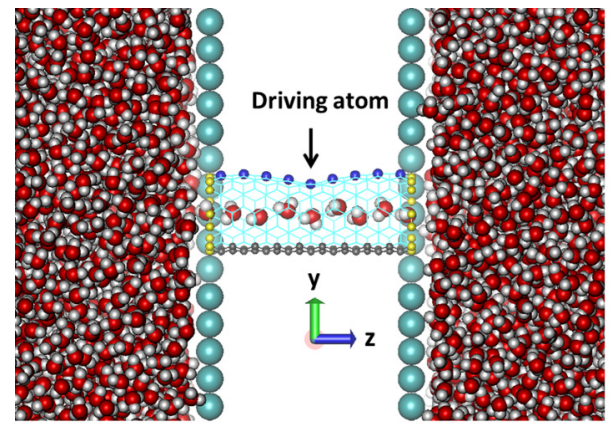

(a)

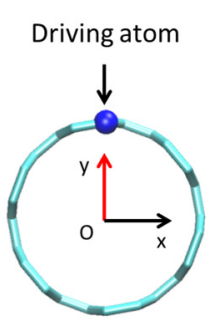

(b)
FIG. 1. (a) Simulation system, consisting of two sheets of fixed carbon atoms serving as a membrane, an uncapped $(6,6)$ single-wall $\mathrm{CNT}$, and water molecules (depicted by spheres with oxygen in red and hydrogen in white). The indicated carbon atom of the CNT was driven to oscillate harmonically along the $y$ axis. The vibrations of some other carbon atoms (blue spheres) are analyzed and described in the text. The carbon atoms (yellow spheres) at the two ends of the CNT were fixed, and the carbon atoms shown in gray spheres were strongly restrained in all simulations. (b) Cross section of the CNT.

harmonic oscillation in the $y$ direction by directly updating its coordinates at every time step. Specifically, the $x$ and $z$ coordinates of the atom are strictly fixed, and the y coordinate follows the motion $y=y_{0}-A \sin (2 \pi f t)$, where $y_{0}$ is the equilibrium position (as shown in Fig. 1), the amplitude $A$ is $0.04 \mathrm{~nm}$ ( $5 \%$ of the diameter), and the vibration frequency $f$ is set to different values in different simulations. We note that the motion of this driving atom is along its radial direction ( $y$ axis; see Fig. 1). In addition, the carbon atoms at the two ends of the CNT are fixed, and the carbon atoms (shown as gray spheres in Fig. 1) diametrically opposite from the driving atom are strongly restrained in all simulations. Other atoms are not directly subject to any artificial manipulation.

All the molecular dynamics simulations were performed by using GROMACS4.0.7 [46] at a constant volume with the periodic lengths of $L_{x}=5.3 \mathrm{~nm}, L_{y}=5.3 \mathrm{~nm}, L_{z}=$ $7.21 \mathrm{~nm}$. A temperature of $300 \mathrm{~K}$ was maintained by using a V-rescale thermostat [47] with $\tau=0.1 \mathrm{ps}$. The TIP3P water model [48] was used, and the carbon atoms in the single-wall CNT (SWCNT) were modeled as uncharged Lennard-Jones particles. The total potential energy function of the SWCNT consists of the standard force field terms:

$$
\begin{aligned}
V_{\text {total }}= & \sum_{\text {VDW }} 4 \varepsilon\left[\left(\frac{\sigma}{r}\right)^{12}-\left(\frac{\sigma}{r}\right)^{6}\right]+\sum_{\text {bond }} \frac{1}{2} k_{b}\left(r-r_{0}\right)^{2} \\
& +\sum_{\text {angles }} \frac{1}{2} k_{\theta}\left(\theta-\theta_{0}\right)^{2}+\sum_{\text {dihedrals }} \frac{1}{2} k_{\xi}\left(\xi-\xi_{0}\right)^{2} .
\end{aligned}
$$

All the parameters are based on Refs. [12,49]: $\sigma_{C C}=$ $0.34 \mathrm{~nm}, \varepsilon_{C C}=0.3612 \mathrm{~kJ} / \mathrm{mol}$, carbon-carbon bond lengths of $r_{0}=0.142 \mathrm{~nm}$, bond angles of $\theta_{0}=120^{\circ}$, spring constants $k_{b}=393960 \mathrm{~kJ} \mathrm{~mol}^{-1} \mathrm{~nm}^{-2}, k_{\theta}=527 \mathrm{~kJ} \mathrm{~mol}^{-1} \mathrm{deg}^{-2}$, and $k_{\xi}=167.434 \mathrm{~kJ} \mathrm{~mol}^{-1} \mathrm{deg}^{-2}$. The long-range electrostatics interactions were handled by using the particle mesh Ewald (PME) method [50] with a cutoff for real space of $1.4 \mathrm{~nm}$. The short-range van der Waals forces also had a cutoff distance of $1.4 \mathrm{~nm}$.
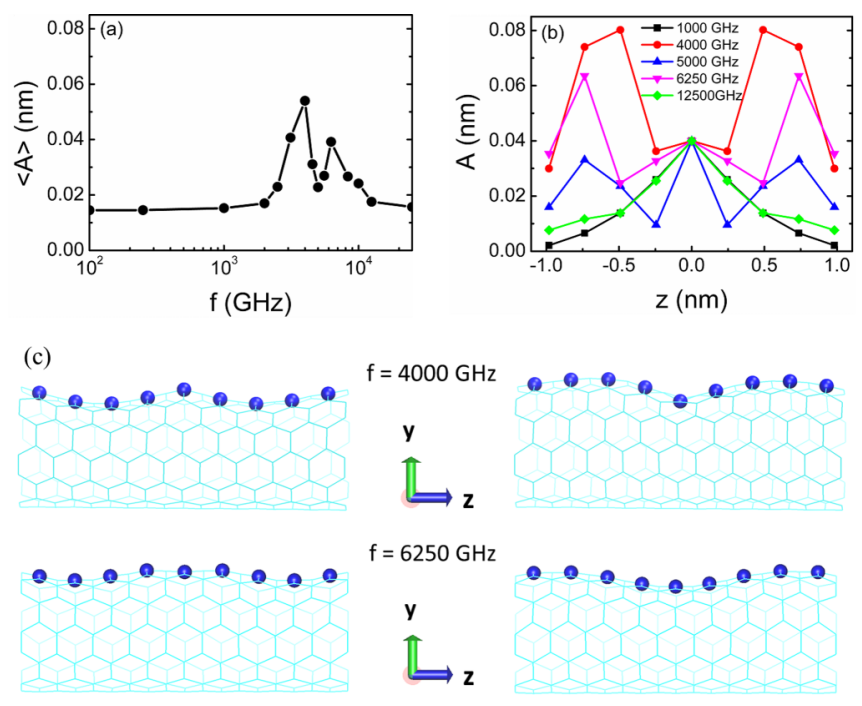

FIG. 2. Vibration of the CNT. (a) Average vibration amplitude over the nine carbon atoms [indicated in panel (c)] in the CNT, in each simulation with a different frequency $f$. (b) Amplitudes of the individual carbon atoms under five different vibration frequencies. (c) Snapshots of the CNT at $f=4000 \mathrm{GHz}$ and $f=6250 \mathrm{GHz}$ with the driving atom at the two extreme positions.

We performed a total of fifteen MD simulations, with the frequency $f$ of the oscillating atom in the range of 100 to $25000 \mathrm{GHz}$. A time step of $0.2 \mathrm{fs}$ was used for simulations at high frequencies ( $\mathrm{f} \geqslant 12500 \mathrm{GHz}$ ), whereas a time step of $1 \mathrm{fs}$ was used for other simulations. Each simulation was run for $25 \mathrm{~ns}$, and the last $20 \mathrm{~ns}$ of the simulation trajectory was used for analysis.

\section{RESULTS AND DISCUSSION}

We first focus on the mechanical vibration of the CNT. In our simulations, as described in Sec. II, one carbon atom in the central plane of the CNT was driven to oscillate harmonically in the radial $(y)$ direction, while the two ends of the CNT as well as some diametrically opposed atoms were fixed in position or restrained (Fig. 1). Consequently, other carbon atoms in the CNT followed the oscillation of the driving atom to various extents. To quantify such vibrations, we took $N$ evenly spaced time points in one full oscillation period of the driving atom, each thus representing a unique phase point. We then selected from the simulation trajectory the frames with the driving atom precisely at a given phase, and calculated the average positions of other carbon atoms over these frames. Therefore, we obtained $N$ average structures for the CNT, each at a unique phase. The $N$ average positions for each individual atom thus delineate its average oscillation cycle, from which the amplitude and other properties for the atom can be subsequently determined.

Figure 2(b) shows the amplitudes for the carbon atoms in the same axial line as the driving atom [corresponding to the nine blue atoms in Fig. 2(c)], in simulations with different oscillation frequencies. As expected, the amplitudes for these atoms are symmetric with respect to the center. By design, the driving (controlled) atom had the same amplitude $(0.04 \mathrm{~nm})$ in 


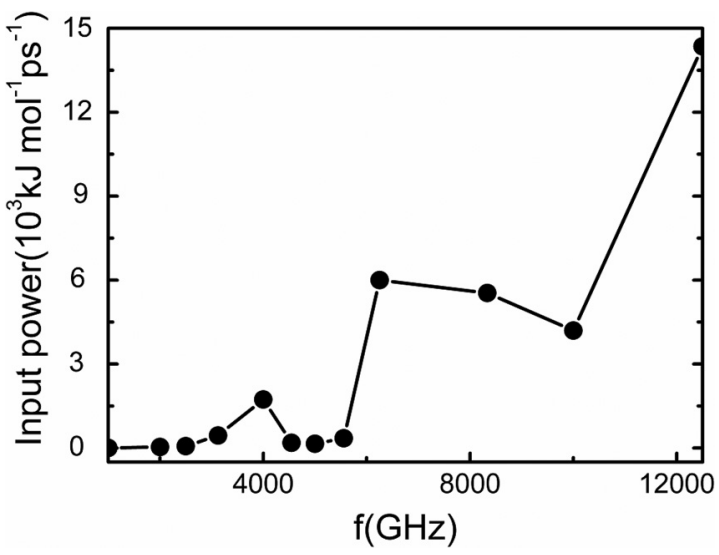

FIG. 3. Average input power for simulations at different vibration frequencies.

all simulations. However, the amplitudes of the other carbon atoms are sensitive to the driving frequency. Figure 2(a) shows the average of these amplitudes in each of the fifteen simulations in this study, which clearly reveals the frequency dependence of the vibration magnitude. At low frequencies $(\leqslant 1000 \mathrm{GHz})$, the amplitudes are relatively small because the carbon atoms have sufficient time to adapt and are thus in instantaneous mechanical equilibrium with the time-varying deformation imposed by the driving atom. At high frequencies $(\geqslant 12500 \mathrm{GHz})$, the oscillations of the carbon atoms also have relatively small magnitudes. In contrast, in the terahertz range, particularly at two "resonance" frequencies (4000 and $6250 \mathrm{GHz}$ ), the CNT exhibited very strong oscillation [Fig. 2(a)], and some carbon atoms vibrated with much larger amplitudes [Fig. 2(b)] than the driving atom did.

By integrating the mechanical work done to the driving atom, we calculated the input power in each simulation (Fig. 3). This external energy input is also equal to the amount of energy dissipated to the thermostat. On average, faster atoms would experience a stronger friction due to the thermostat and thus dissipate more energy. Under similar amplitudes, vibrations at higher frequencies have larger average speeds and therefore consume more energy, thus resulting in an overall increase in the input power (Fig. 3) with driving frequency. Furthermore, as expected, the input power depends on the temperature-coupling parameter in the simulations and becomes smaller under weaker coupling (i.e., a larger value of $\tau)$ to the thermostat.

To evaluate the influence of water on the motion of the CNT, we performed simulations of the CNT in vacuum (without water) at two driving frequencies (1000 and $4000 \mathrm{GHz})$. The behavior of the CNT in vacuum turned out to be almost identical (Fig. S1) [51] to those in the simulations of the CNT in water, thus indicating that water has little effect on the CNT vibration. This may be related to the large rate of energy input and dissipation (Fig. 3) by the CNT in these particular simulations, which make the friction and damping due to water relatively insignificant. In such cases, therefore, the frequency dependence appears to be intrinsic properties of the CNT, and independent of water. Earlier theoretical and experimental works also showed that the resonance frequency
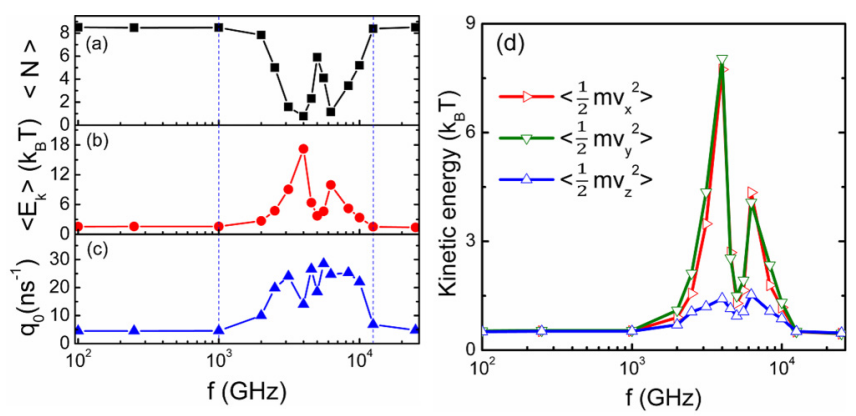

FIG. 4. (a) Average number of water molecules inside the CNT in each simulation with a different vibration frequency. (b) Average translational kinetic energy of the water molecules. (c) Average number of permeation events in one direction per unit time. (d) Average contribution of each velocity component $\left(v_{x}, v_{y}, v_{z}\right)$ to the kinetic energy.

of a CNT sensitively depends on its own structural and mechanical properties [52-54].

If the CNT could be modeled as an ideal one-dimensional continuum medium, the induced oscillation would be in the form of a standing wave. However, the continuum model is clearly oversimplified here, given that our CNT is relatively short, with only $\sim 10$ carbon atoms along each axial line. In addition, the vibrations are not in one dimension only. Furthermore, damping due to the thermostat as well as water will result in deviations from the ideal standing wave. Nonetheless, the oscillation pattern of the CNT [illustrated in Fig. 2(c), with the driving atom at the two extremities] is qualitatively reminiscent of a standing wave.

Next, we examine the behavior of water molecules. Figure 4(a) and Table S1 [51] show the average number $\langle N\rangle$ of water molecules inside the CNT in each simulation. At low or high frequencies $(\leqslant 1000 \mathrm{GHz}$ or $\geqslant 12500 \mathrm{GHz})$, the $\mathrm{CNT}$ is, on average, occupied by 8.5 water molecules, which are normally aligned in a single file (Fig. 1), as observed in many equilibrium simulations of CNTs (without the active vibration) $[12,28,55]$. At resonance frequencies, in contrast, the average water occupancy drops dramatically (to about 0.8 and 1.2 at $f=4000 \mathrm{GHz}$ and $f=6250 \mathrm{GHz}$, respectively), indicating that the water chain inside the CNT was completely broken. Furthermore, the frequency dependence of the water occupancy [Fig. 4(a)] is clearly anticorrelated with the vibration amplitudes [Fig. 2(a)] of the CNT.

To evaluate water mobility, we counted the number of permeation events (defined as a water molecule entering the CNT at one end and exiting at the other end) in each simulation. Due to the symmetry of the CNT, we do not expect a net water flux even in these nonequilibrium simulations. Indeed, our observed permeation events in the two directions have roughly equal counts (Table S1) [51]. Figure 4(c) shows the average number of permeation events in one direction per unit time, denoted $q_{0}$, for each simulation. $q_{0}$ quantifies the rate of water exchange between the two sides and is directly related to the diffusion permeability of the channel [56]. At high and low frequencies, the water exchange rate $q_{0}$ is similar to the value in equilibrium simulations. For the frequencies at which the CNT vibrates in large amplitudes; however, $q_{0}$ is also significantly enhanced [Fig. 4(c)], even in the simulations (at 
the resonance frequencies) with very low water occupancies [Fig. 4(a)] in the CNT.

Intuitively, the low water occupancies [Fig. 4(a)] in the strongly-vibrating CNT might result simply from the mechanical deformation. To evaluate such effects, we first identified the two extremities for the atomic positions [Fig. 2(c)] when the CNT vibrates at the resonance frequency of $4000 \mathrm{GHz}$, and then performed two simulations in which all CNT atoms were fixed at these extreme positions. In the simulations that reproduced the maximum deformations but without the dynamic vibration of the CNT, we observed normal water occupancy (Fig. S2) [51] and no enhancement of the water mobility. Therefore, the static deformation alone (with the magnitudes here) cannot account for the dramatic changes in water occupancy and mobility in the vibrating CNT. However, note that, at sufficiently large deformations, the CNT will become empty, as reported by Andreev et al. [57].

With static deformation unlikely to be the primary mechanism for the abnormal water behavior, we then examined the heating effect in the vibrating CNT. Figure 4(b) shows the average (translational) kinetic energy (in units of $k_{B} T$, where $k_{B}$ is the Boltzmann constant and $T=300 \mathrm{~K}$ is the temperature of the thermal bath) for the water molecules in the CNT, determined by their center-of-mass velocities. These water kinetic energies [Fig. 4(b)] are also highly correlated with the oscillation amplitudes of the CNT [Fig. 2(a)], which thus appear to be the major determinant of the unusual water behavior. At low or high frequencies, the average kinetic energy is about $\frac{3}{2} k_{B} T$, which is consistent with the equipartition theorem valid for equilibrium systems. Therefore, the water molecules in these simulations did not significantly deviate from equilibrium or were affected by the small-scale vibration of the CNT. In contrast, at the resonance frequencies, the kinetic energy (17.2 $k_{B} T$ at $\left.f=4000 \mathrm{GHz}\right)$ exceeds the equilibrium value by an order of magnitude and would correspond to a temperature of $3000 \mathrm{~K}$ in equilibrium systems. Therefore, when the CNT was strongly vibrating at the resonance frequencies, the few water molecules inside were moving at high speeds. Consequently, the continuous water chain (as in the equilibrium systems) was completely disrupted, thus preventing the accommodation of more water molecules in the CNT and resulting in a very low occupancy [Fig. 4(a)]. Furthermore, Figure 4(d) shows that the velocity components ( $x$ and $y$ ) perpendicular to the CNT axis are much larger than the axial $(z)$ component, thus indicating that the fast motions of the water molecules are primarily in the $x-y$ plane.

Our simulation trajectories also revealed how the kinetic energy is transferred to the water molecules inside the CNT. Figure 5 provides the details of representative permeation events in the simulations with driving frequencies of $4000 \mathrm{GHz}(T=0.25 \mathrm{ps})$ and $12500 \mathrm{GHz}(T=0.08 \mathrm{ps})$. The trajectory at $f=4000 \mathrm{GHz}$ [Fig. 5(c)] shows that a water molecule underwent fast and strong oscillations after entering the empty CNT. Its velocity [Fig. 5(e)] in the $x$ and $y$ directions had root-mean-square values of about $2.1 \times 10^{3} \mathrm{~m} / \mathrm{s}$, in comparison with $\sim 400 \mathrm{~m} / \mathrm{s}$ in equilibrium systems. Interestingly, the trajectory [Fig. 5(c)] of its $z$ coordinate indicates that, within the short duration ( $27 \mathrm{ps)}$ of this permeation event, the water molecule bounced back and forth several times between the two ends before completely exiting the CNT. It (a)
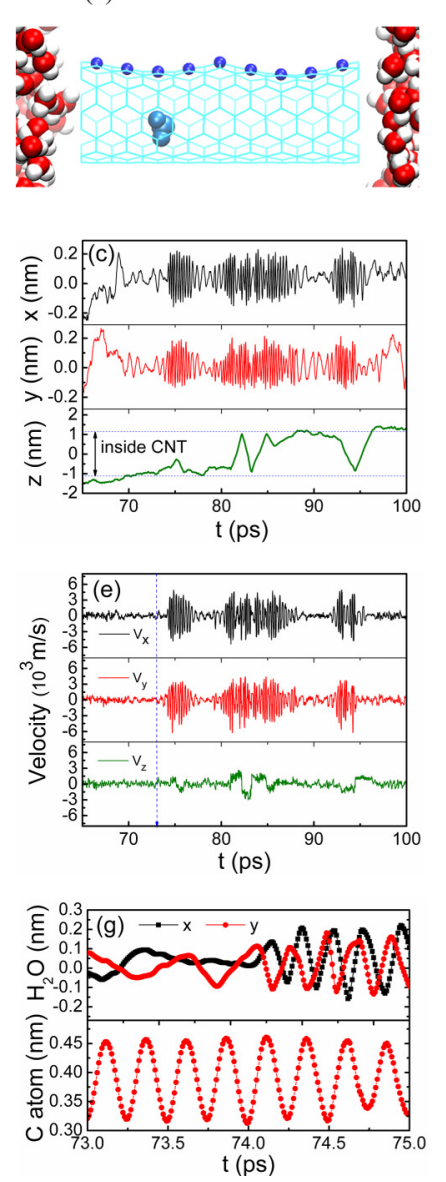

(b)
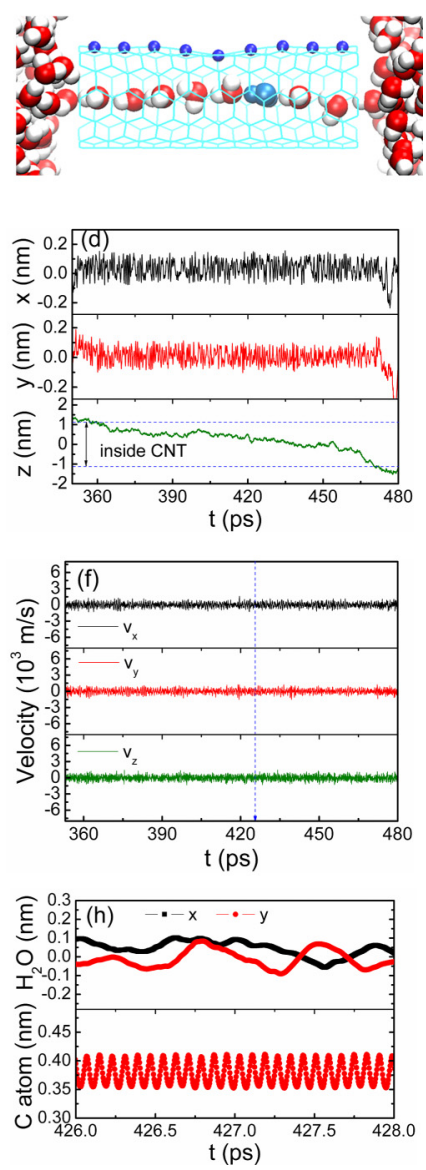

FIG. 5. Motion of a labeled water molecule (represented by its oxygen atom) inside the $\mathrm{CNT}$ in the simulations at vibration frequencies of 4000 and $12500 \mathrm{GHz}$. (a), (b) Snapshots of the CNT and nearby water molecules, with the labeled water molecule shown in blue. (c), (d) Position of the labeled water molecule when it crossed the CNT. (e), (f) Instantaneous velocity of the water molecule during the same period. (g), (h) The $x$ and $y$ coordinates of the water molecule and the $y$ coordinate of a nearby carbon atom during a period of 2 ps starting at the time indicated by the dashed arrows in panels (e) and (f).

appears that, when the water molecule approaches the entrance of the CNT with a high speed, it may instantly reverse its velocity [Fig. 5(e)] due to an almost-elastic collision with the bulk water. Furthermore, a closer look at the trajectory shows that, shortly after the water molecule entered the CNT, it gained large velocities (especially in the $x-y$ plane) by colliding with the strongly vibrating carbon atoms. While inside the CNT, the water molecule oscillated at essentially the same frequency as the carbon atoms [Fig. 5(g)]. Similar motions of the water molecules in the CNT were also observed in the simulation at the other resonance frequency $f=6250 \mathrm{GHz}$ (see Fig. S3). [51] Therefore, despite an almost empty CNT [Figs. 4(a) and 5(a)] at the resonance frequencies, the very fast kinetics of individual water molecules nonetheless enables the relatively large water exchange rates [Fig. 4(c)].

At high frequency $f=12500 \mathrm{GHz}$, in contrast, although the carbon atom [Fig. 5(h)] had a large average speed $(1250 \mathrm{~m} / \mathrm{s})$, the water molecules in the CNT were almost 
unaffected, most likely because of the small vibration amplitudes of the carbon atoms [Figs. 5(h) and 2(b)]. In this simulation, the water molecules behaved much like in the equilibrium systems, with normal velocities [Fig. 5(f)] and typical single-file [Fig. 5(b)] diffusions. Therefore, vibrations of the CNT atoms with sufficiently large amplitudes appear to be necessary to produce the abnormal water behavior as in the simulations at the resonance frequencies. We also note that, for narrow channels, previous simulations by Lu et al. [58] and Falk et al. [29] showed that small variations in the size of the pore could significantly alter the water behavior.

As mentioned before, the coupling to the thermostat has a significant effect on the energy input and dissipation of the CNT. We further examined the effects on other behaviors by repeating several simulations with a weaker thermal coupling ( $\tau=0.5 \mathrm{ps}$ ). A comparison of the simulations indicated that the thermal coupling only has substantial effects on the vibration amplitudes for simulations at high frequencies (12500 GHz or higher). Furthermore, the water occupancy and kinetic energy are very similar in the simulations with strong and weak thermal couplings. Therefore, the main conclusion of this study would not be significantly affected by the choice of the coupling parameter $\tau$. In addition, under the thermal coupling parameters tested here, the majority of the input energy is directly dissipated by the CNT to the thermostat, and no substantial heating of the bulk water is observed in the simulations.

\section{CONCLUSIONS}

Our simulations and analysis revealed the underlying physical mechanism for the effect of controlled vibration of a CNT.
When one atom of the CNT is artificially driven to oscillate radially while the two ends are fixed, the vibration pattern of the CNT is mainly determined by its intrinsic properties, regardless of the presence of water. At resonance frequencies, the amplitudes of some carbon atoms of the CNT far exceed that of the driving atom. In such cases the water molecules inside the CNT will be significantly affected. On the one hand, the continuous $\mathrm{H}$-bonded water chain will be disrupted, thus dramatically reducing the number of water molecules in the CNT. On the other hand, water molecules that enter the CNT could gain a very high speed due to collisions with the vibrating carbon atoms and acquire a very large kinetic energy that they would be unlikely to have under equilibrium conditions. When the CNT vibrates at large amplitudes, therefore, the water mobility and the kinetic rate of water exchange are also significantly enhanced, even under low water occupancies in the CNT.

We note that the simulation setup here is certainly not the best representation of real experiments. Instead, the main objective of this study is to elucidate the physical mechanisms in this highly idealized model system. The fundamental understanding gained from this study should be helpful for improving the design of similar simulations in future studies and should be a first step toward the rational design of nanodevices.

\section{ACKNOWLEDGMENTS}

This work was supported by the National Natural Science Foundation of China under Grants No. 11505156 and No. 11875237 and the China Scholarship Council under Grant No. 201707260004. We thank H. Meshkin for technical assistance.
[1] K. Murata, K. Mitsuoka, T. Hirai, T. Walz, P. Agre, J. B. Heymann, A. Engel, and Y. Fujiyoshi, Nature (London) 407, 599 (2000).

[2] B. L. de Groot and H. Grubmuller, Science 294, 2353 (2001).

[3] M. Whitby and N. Quirke, Nat. Nanotech. 2, 87 (2007).

[4] H. G. Park and Y. Jung, Chem. Soc. Rev. 43, 565 (2014).

[5] A. Kalra, S. Garde, and G. Hummer, Proc. Natl. Acad. Sci. USA 100, 10175 (2003).

[6] F. Q. Zhu, E. Tajkhorshid, and K. Schulten, Phys. Rev. Lett. 93, 224501 (2004).

[7] M. Majumder, N. Chopra, R. Andrews, and B. J. Hinds, Nature (London) 438, 44 (2005).

[8] J. K. Holt, H. G. Park, Y. M. Wang, M. Stadermann, A. B. Artyukhin, C. P. Grigoropoulos, A. Noy, and O. Bakajin, Science 312, 1034 (2006).

[9] S. Joseph and N. R. Aluru, Nano Lett. 8, 452 (2008).

[10] J. A. Thomas and A. J. H. McGaughey, Phys. Rev. Lett. 102, 184502 (2009).

[11] S. K. Kannam, B. D. Todd, J. S. Hansen, and P. J. Daivis, J. Chem. Phys. 138, 094701 (2013).

[12] G. Hummer, J. C. Rasaiah, and J. P. Noworyta, Nature (London) 414, 188 (2001).

[13] R. Allen, S. Melchionna, and J. P. Hansen, Phys. Rev. Lett. 89, 175502 (2002).
[14] O. Beckstein and M. S. P. Sansom, Proc. Natl. Acad. Sci. USA 100, 7063 (2003).

[15] H. J. Lu, X. Y. Zhou, F. M. Wu, and Y. S. Xu, J. Phys. Chem. B 112, 16777 (2008).

[16] J. Dzubiella and J. P. Hansen, J. Chem. Phys. 122, 234706 (2005).

[17] O. Beckstein, P. C. Biggin, and M. S. P. Sansom, J. Phys. Chem. B 105, 12902 (2001).

[18] R. Z. Wan, J. Y. Li, H. J. Lu, and H. P. Fang, J. Am. Chem. Soc. 127, 7166 (2005).

[19] J. Y. Li, X. J. Gong, H. J. Lu, D. Li, H. P. Fang, and R. H. Zhou, Proc. Natl. Acad. Sci. USA 104, 3687 (2007).

[20] E. Tajkhorshid, P. Nollert, M. O. Jensen, L. J. W. Miercke, J. O'Connell, R. M. Stroud, and K. Schulten, Science 296, 525 (2002).

[21] B. Corry, Energy Environ. Sci. 4, 751 (2011).

[22] R. Das, M. E. Ali, S. B. Abd Hamid, S. Ramakrishna, and Z. Z. Chowdhury, Desalination 336, 97 (2014).

[23] P. Král and M. Shapiro, Phys. Rev. Lett. 86, 131 (2001).

[24] S. Ghosh, A. K. Sood, and N. Kumar, Science 299, 1042 (2003).

[25] Q. Yuan and Y.-P. Zhao, J. Am. Chem. Soc. 131, 6374 (2009).

[26] Y. Zhao et al., Adv. Mater. 20, 1772 (2008).

[27] W. Yang, P. Thordarson, J. J. Gooding, S. P. Ringer, and F. Braet, Nanotechnol. 18, 412001 (2007). 
[28] A. Berezhkovskii and G. Hummer, Phys. Rev. Lett. 89, 064503 (2002).

[29] K. Falk, F. Sedlmeier, L. Joly, R. R. Netz, and L. Bocquet, Nano Lett. 10, 4067 (2010).

[30] E. Secchi, S. Marbach, A. Nigues, D. Stein, A. Siria, and L. Bocquet, Nature (London) 537, 210 (2016).

[31] L. Bocquet and J. L. Barrat, J. Chem. Phys. 139, 044704 (2013).

[32] L. Bocquet and E. Charlaix, Chem. Soc. Rev. 39, 1073 (2010).

[33] E. Yamamoto, T. Akimoto, Y. Hirano, M. Yasui, and K. Yasuoka, Phys. Rev. E 89, 022718 (2014).

[34] I. Kosztin and K. Schulten, Phys. Rev. Lett. 93, 238102 (2004).

[35] W. Im and B. Roux, J. Mol. Biol. 322, 851 (2002).

[36] Z. Insepov, D. Wolf, and A. Hassanein, Nano Lett. 6, 1893 (2006).

[37] H. Qiu, R. Shen, and W. L. Guo, Nano Res. 4, 284 (2011).

[38] X. P. Li, G. P. Kong, X. Zhang, and G. W. He, Appl. Phys. Lett. 103, 143117 (2013).

[39] Q. L. Zhang, W. Z. Jiang, J. Liu, R. D. Miao, and N. Sheng, Phys. Rev. Lett. 110, 254501 (2013).

[40] W. H. Duan and Q. Wang, ACS Nano 4, 2338 (2010).

[41] M. Khodabakhshi and A. Moosavi, J. Phys. Chem. C 121, 23649 (2017).

[42] H. J. Lu, X. J. Gong, C. L. Wang, H. P. Fang, and R. Z. Wang, Chin. Phys. Lett. 25, 1145 (2008).

[43] H. J. Lu, X. C. Nie, F. M. Wu, X. Y. Zhou, and J. L. Kou, J. Chem. Phys. 136, 174511 (2012).

[44] J. L. Kou, H. J. Lu, F. M. Wu, J. T. Fan, and J. Yao, Nano Lett. 14, 4931 (2014).
[45] X. Y. Zhou, F. M. Wu, Y. Liu, J. L. Kou, H. Lu, and H. J. Lu, Phys. Rev. E 92, 053017 (2015).

[46] B. Hess, C. Kutzner, D. van der Spoel, and E. Lindahl, J. Chem. Theory Comput. 4, 435 (2008).

[47] G. Bussi, D. Donadio, and M. Parrinello, J. Chem. Phys. 126, 014101 (2007).

[48] W. L. Jorgensen, J. Chandrasekhar, J. D. Madura, R. W. Impey, and M. L. Klein, J. Chem. Phys. 79, 926 (1983).

[49] W. D. Cornell, P. Cieplak, C. I. Bayly, I. R. Gould, K. M. Merz, D. M. Ferguson, D. C. Spellmeyer, J. W. Caldwell, and P. A. Kollman, J. Am. Chem. Soc. 117, 5179 (1995).

[50] T. Darden, D. York, and L. Pedersen, J. Chem. Phys. 98, 10089 (1993).

[51] See Supplemental Material at http://link.aps.org/supplemental/ 10.1103/PhysRevE.98.032410 for Table S1 and Figs. S1-S3.

[52] V. Sazonova, Y. Yaish, H. Üstünel, D. Roundy, T. A. Arias, and P. L. McEuen, Nature (London) 431, 284 (2004).

[53] R. S. A. Jorio, J. H. Hafner, C. M. Lieber, M. Hunter, T. McClure, G. Dresselhaus, and M. S. Dresselhaus, Phys. Rev. Lett. 86, 1118 (2001).

[54] C. Y. Li and T. W. Chou, Phys. Rev. B 68, 073405 (2003).

[55] F. Q. Zhu and K. Schulten, Biophys J 85, 236 (2003).

[56] F. Q. Zhu, E. Tajkhorshid, and K. Schulten, Biophys J 86, 50 (2004).

[57] S. Andreev, D. R. Reichman, and G. Hummer, J. Chem. Phys. 123, 194502 (2005).

[58] H. J. Lu, J. Y. Li, X. J. Gong, R. Z. Wan, L. Zeng, and H. P. Fang, Phys. Rev. B 77, 174115 (2008). 